\title{
Influência de Diferentes Dietas em Fases Imaturas de Ceraeochrysa cubana (Hagen) (Neuroptera: Chrysopidae)
}

\author{
Lenira V.C. Santa-Cecília ${ }^{1}$, Brígida Souza ${ }^{2}$ e César F. Carvalho ${ }^{2}$ \\ ${ }^{1}$ EPAMIG/CRSM, Caixa postal 176, 37200-000, Lavras, MG. \\ ${ }^{2}$ Universidade Federal de Lavras, Departamento de Entomologia, \\ Caixa postal 37, 37200-000, Lavras, MG.
}

An. Soc. Entomol. Brasil 26(2): 309-314 (1997)

\begin{abstract}
Effects of Different Diets on the Immature Stages of Ceraeochrysa cubana (Hagen) (Neuroptera: Chrysopidae)
\end{abstract}

\begin{abstract}
Larval and pupal development of the predator Ceraeochrysa cubana (Hagen) on different diets was evaluated. Larvae (20/treatment), were reared in laboratory at $20 \pm 2{ }^{\circ} \mathrm{C}, 70 \pm 10 \% \mathrm{RH}$ and 12 hours photophase on the following diets; eggs of Anagasta kuehniella; Toxoptera sp.; Pinnaspis sp.; eggs of A. kuehniella + Toxoptera sp.; eggs of A. kuehniella + Pinnaspis sp.; Toxoptera sp. + Pinnaspis sp.; and eggs of A. kuehniella + Toxoptera sp. + Pinnaspis sp. In all treatments, larvae fed on $A$. kuehniella eggs, showed shorter larval and pupal duration, higher number of adults, with viability of 75, 70, 95 and $80 \%$ for treatments eggs of A. kuehniella, eggs of A. kuehniella + Toxoptera sp.; eggs of A. kuehniella + Pinnaspis sp., respectively. The diets with Toxoptera sp., Pinnaspis sp. and eggs of Toxoptera sp. + Pinnaspis sp. were the least suitable for the development of the predator with viability as low as $0 ; 5$; and $15 \%$, respectively.
\end{abstract}

KEY WORDS: Insecta, green lacewing, predator, diet, development.

RESUMO - O desenvolvimento larval e pupal de Ceraeochrysa cubana em diferentes dietas foi estudado. As larvas (20 por tratamento), foram criadas em laboratório a $25 \pm 2{ }^{\circ} \mathrm{C}, 70 \pm 10 \%$ UR e fotofase de 12 horas, nas seguintes dietas: ovos de Anagasta kuehniella; Toxoptera sp.; Pinnaspis sp.; ovos de A. kuehniella + Toxoptera sp.; ovos de A. kuehniella + Pinnaspis sp.; Toxoptera sp. + Pinnaspis sp.; ovos de A. kuehniella + Toxoptera sp. + Toxoptera sp. As larvas que receberam ovos de A. kuehniella, em todos os tratamentos, apresentaram menor duração das fases larval e pupal e permitiram a obtenção de maior número de adultos com 75, 70, 95 e $80 \%$ de viabilidade para os tratamentos com ovos A. kuehniella, ovos de de A. kuehniella + Toxoptera sp., ovos de A. kuehniella + Toxoptera sp. + Pinnaspis sp., respectivamente. Dietas com Toxoptera sp., Pinnaspis sp. e Toxoptera sp. + Pinnaspis sp. foram inadequadas para o desenvolvimento do predador com viabilidade de $0 ; 5$ e 15 $\%$, respectivamente.

PALAVRAS-CHAVE: Insecta, predador, crisopídeo, dieta. 
Os crisopídeos são predadores polífagos encontrados em muitas culturas de interesse econômico, exercendo importante papel no controle biológico de artrópodos fitófagos (Tauber 1974, Agnew et al. 1981, Adams \& Penny 1985). Dentre as espécies que ocorrem com maior frequência em pomares cítricos na região de Lavras-MG, Ceraeochrysa cubana (Hagen) apresenta potencial para uso em programas de manejo integrado de pragas (Venzon \& Carvalho 1993). A alimentação da fase larval tem sido o maior obstáculo para sua criação em laboratório. Obrycki et al. (1989) mencionaram que a adequação de presas para predadores polífagos é variável. Muma (1957) investigando os efeitos da nutrição no ciclo de vida de Chrysopa lateralis Guer., constatou que, embora algumas presas fossem aceitas pelas larvas, foram impróprias ao seu desenvolvimento. Ribeiro (1988) verificou que larvas de Chrysoperla externa (Hagen) alimentadas com o pulgão Aphis gossypii Glover (Homoptera: Aphididae) completaram o desenvolvimento em 10,3 dias com 93,3\% de viabilidade, enquanto que, quando alimentadas com o pulgão Toxoptera citricidus Kirkaldy (Homoptera: Aphididae), as larvas não sobreviveram além do $2^{2}$ ínstar. Moraes (1989) também observou que larvas de $C$. cubana, alimentadas com Toxoptera sp., não completavam esta fase do desenvolvimento, morrendo durante o 3 o ínstar. Pasqualini (1975) verificou que larvas de Chrysoperla carnea (Stephens), alimentadas com ovos de Anagasta kuehniella (Zeller) (Lepidoptera: Pyralidae), tiveram duração de 15 dias, com $73,5 \%$ de casulos formados. Entretanto, para larvas alimentadas com lagartas, a duração aumentou para 21 dias, com 69,2\% de pupação.

Este estudo teve como objetivo verificar o desenvolvimento larval e pupal de $C$. cubana em diferentes dietas.

\section{Material e Métodos}

O trabalho foi conduzido no laboratório de Biologia de Insetos do Departamento de
Entomologia da Universidade Federal de Lavras, à $25 \pm 2{ }^{\circ} \mathrm{C}$, UR de $70 \pm 10 \%$ e fotofase de 12 horas. Os ovos de A. kuehniella utilizados foram provenientes de criação laboratorial e os pulgões e cochonilhas foram coletados diretamente em laranjeiras (Citrus sinensis) em área experimental. Ovos de $C$. cubana foram individualizados em tubos de vidro $(2,5 \times 8,5 \mathrm{~cm})$ e fechados com filme de polietileno. As larvas foram alimentadas desde a eclosão até completo desenvolvimento com sete dietas diferentes: ovos de A. kuehniella; Toxoptera sp.; Pinnaspis sp.; ovos de A. kuehniella + Toxoptera sp.; ovos de A. kuehniella + Pinnaspis sp.; Toxoptera sp. + Pinnaspis sp.; ovos de A. kuehniella + Toxoptera sp. + Pinnaspis sp.. Ovos de A. kuehniella e o pulgão Toxoptera sp. foram colocados no interior dos tubos de vidro, com auxílio de um pincel. A cochonilha Pinnaspis sp. foi fornecida em secções recortadas de folhas de citros, colonizadas com os insetos.

Os tratamentos foram repetidos 20 vezes, em delineamento inteiramente casualizado, sendo cada parcela experimental constituída por uma larva do predador. As avaliações foram feitas diariamente, desde a eclosão até a emergência dos adultos, observando-se os seguintes parâmetros: número de ínstares, duração e viabilidade de cada ínstar e da fase pupal. Os dados relativos à duração de cada ínstar e da fase pupal foram analisados pelo teste $\mathrm{F}$, sendo as médias comparadas pelo teste de Tukey, ao nível de $5 \%$ de probabilidade.

\section{Resultados e Discussão}

Duração dos Ínstares. Constatou-se que os diferentes tipos de presa testados afetaram significativamente a duração dos três ínstares (Tabela 1). Larvas alimentadas apenas com ovos de A. kuehniella apresentaram duração menor do 1 o ínstar, enquanto que aquelas que receberam apenas Pinnaspis sp. tiveram duração maior. As demais dietas proporcionaram duração intermediária, não diferindo significativamente entre si, com variação de 4,6 a 5,1 dias. Esses resultados concordam com os obtidos por Moraes 
(1989), que também não encontrou diferenças significativas na duração do 1ํ ínstar, quando alimentou larvas de C. cubana com diferentes dietas contendo ovos de A. kuehniella. combinação destes com Toxoptera sp. ou Pinnaspis sp.. A maior duração desse ínstar foi observada para as larvas alimentadas com Pinnaspis sp. ou Toxoptera sp. + Pinnaspis

Tabela 1. Duração $(\mathrm{X} \pm \mathrm{EP})$, em dias, das fases imaturas de Ceraeochrysa cubana em dietas diferentes.

\begin{tabular}{|c|c|c|c|c|c|c|}
\hline \multirow{3}{*}{ Tratamentos } & \multicolumn{5}{|c|}{ Duração $^{1}$} & \multirow{3}{*}{$\begin{array}{c}\text { Período } \\
\text { (larva a } \\
\text { adulto) }\end{array}$} \\
\hline & \multicolumn{3}{|c|}{ Ínstares } & \multicolumn{2}{|c|}{ Fase } & \\
\hline & $1^{\mathrm{o}}$ & $2^{-}$ & $3^{\circ}$ & Larva & Pupa & \\
\hline 1. A. kuehniella & $\begin{array}{c}3,8 \mathrm{c} \\
( \pm 0,6) \\
{[20]}\end{array}$ & $\begin{array}{c}4,0 \mathrm{c} \\
( \pm 0,0) \\
{[20]}\end{array}$ & $\begin{array}{c}4,9 \mathrm{~d} \\
( \pm 0,4) \\
{[20]}\end{array}$ & $\begin{array}{c}12,7 \mathrm{e} \\
( \pm 0,5) \\
{[20]}\end{array}$ & $\begin{array}{c}12,9 \text { a } \\
( \pm 0,8) \\
{[15]}\end{array}$ & $\begin{array}{c}25,7 \mathrm{~d} \\
( \pm 1,0) \\
{[15]}\end{array}$ \\
\hline 2. Toxoptera sp. & $\begin{array}{c}4,9 \mathrm{~b} \\
( \pm 0,5) \\
{[20]}\end{array}$ & $\begin{array}{c}3,9 \mathrm{c} \\
( \pm 0,5) \\
{[19]}\end{array}$ & $\begin{array}{c}10,2 \text { a } \\
( \pm 1,2) \\
{[6]}\end{array}$ & $\begin{array}{c}19,0 \mathrm{~b} \\
( \pm 1,1) \\
{[6]}\end{array}$ & $\begin{array}{l}- \\
- \\
-\end{array}$ & $\begin{array}{l}- \\
- \\
-\end{array}$ \\
\hline 3. Pinnaspis sp. & $\begin{array}{c}6,5 \mathrm{a} \\
( \pm 0,7) \\
{[19]}\end{array}$ & $\begin{array}{c}5,5 \mathrm{a} \\
( \pm 0,8) \\
{[13]}\end{array}$ & $\begin{array}{c}8,5 \mathrm{~b} \\
( \pm 4,9) \\
{[2]}\end{array}$ & $\begin{array}{c}20,5 \text { a } \\
( \pm 4,9) \\
{[2]}\end{array}$ & $\begin{array}{c}13,0 \mathrm{a} \\
( \pm 0,0) \\
{[1]}\end{array}$ & $\begin{array}{c}28,0 \mathrm{~b} \\
( \pm 0,0) \\
{[1]}\end{array}$ \\
\hline $\begin{array}{l}\text { 4. A. kuehniella }+ \\
\text { Toxoptera } \mathrm{sp} .\end{array}$ & $\begin{array}{c}5,1 \mathrm{~b} \\
( \pm 0,5) \\
{[20]}\end{array}$ & $\begin{array}{c}4,0 \mathrm{c} \\
( \pm 0,0) \\
{[16]}\end{array}$ & $\begin{array}{r}4,3 \text { de } \\
( \pm 0,8) \\
{[14]}\end{array}$ & $\begin{array}{c}13,4 \text { e } \\
( \pm 1,3) \\
{[14]}\end{array}$ & $\begin{array}{c}11,5 \mathrm{~b} \\
( \pm 1,3) \\
{[14]}\end{array}$ & $\begin{array}{c}24,9 \mathrm{~d} \\
( \pm 1,7) \\
{[14]}\end{array}$ \\
\hline $\begin{array}{l}\text { 5. A. kuehniella }+ \\
\text { Pinnaspis sp. }\end{array}$ & $\begin{array}{c}5,0 \mathrm{~b} \\
( \pm 0,0) \\
{[20]}\end{array}$ & $\begin{array}{c}4,4 \mathrm{bc} \\
( \pm 0,5) \\
{[20]}\end{array}$ & $\begin{array}{r}4,0 \text { e } \\
( \pm 0,2) \\
{[19]}\end{array}$ & $\begin{array}{c}13,5 \text { de } \\
( \pm 0,6) \\
{[19]}\end{array}$ & $\begin{array}{c}11,6 \mathrm{~b} \\
( \pm 1,4) \\
{[19]}\end{array}$ & $\begin{array}{c}25,1 \mathrm{~d} \\
( \pm 1,5) \\
{[19]}\end{array}$ \\
\hline $\begin{array}{l}\text { 6. Toxoptera sp.+ } \\
\text { Pinnaspis sp. }\end{array}$ & $\begin{array}{c}4,6 \mathrm{~b} \\
( \pm 0,7) \\
{[20]}\end{array}$ & $\begin{array}{c}4,9 \mathrm{ab} \\
( \pm 0,4) \\
{[20]}\end{array}$ & $\begin{array}{r}7,5 \mathrm{c} \\
( \pm 0,7) \\
{[10]}\end{array}$ & $\begin{array}{c}17,1 \mathrm{c} \\
( \pm 0,9) \\
{[10]}\end{array}$ & $\begin{array}{c}13,3 \text { a } \\
( \pm 1,2) \\
{[3]}\end{array}$ & $\begin{array}{c}30,0 \text { a } \\
( \pm 0,0) \\
{[19]}\end{array}$ \\
\hline $\begin{array}{l}\text { 7. A. kuehniella + } \\
\text { Toxoptera } \mathrm{sp} .+ \\
\text { Pinnaspis sp. }\end{array}$ & $\begin{array}{c}5,0 \mathrm{~b} \\
( \pm 0,5) \\
{[20]}\end{array}$ & $\begin{array}{c}4,6 \mathrm{~b} \\
( \pm 1,3) \\
{[20]} \\
\end{array}$ & $\begin{array}{r}4,7 \mathrm{de} \\
( \pm 1,4) \\
{[18]} \\
\end{array}$ & $\begin{array}{c}14,1 \mathrm{~d} \\
( \pm 1,6) \\
{[18]}\end{array}$ & $\begin{array}{c}12,4 \mathrm{ab} \\
( \pm 1,9) \\
{[16]}\end{array}$ & $\begin{array}{c}26,8 \mathrm{c} \\
( \pm 1,8) \\
{[16]}\end{array}$ \\
\hline $\mathrm{CV}(\%)$ & 10,8 & 13,6 & 12,5 & 3,5 & 8,4 & 4,2 \\
\hline
\end{tabular}

${ }^{1}$ Médias seguidas pela mesma letra não diferem entre si pelo teste de Tukey, ao nível de $5 \%$ de probabilidade.

Para o $2^{\circ}$ ínstar, a menor duração ocorreu quando as larvas foram alimentadas com Toxoptera sp., com ovos de A. kuehniella e a sp.. Os resultados obtidos são concordantes com os de Moraes (1989), verificados para C. cubana, com os de Awadallah et al. (1975), 
para C. carnea, os de Ribeiro (1988) para $C$. externa e os de Obrycki et al. (1989), para $C$. carnea e $C$. oculata Say, os quais constataram que a dieta fornecida às larvas interferiu no desenvolvimento dessa fase, especialmente no 2o ínstar. No entanto, esses resultados divergiram dos obtidos para C. cubana, por Venzon \& Carvalho (1993), que não observaram diferenças entre os ínstares, em função da alimentação. Isto pode ser atribuído à adaptabilidade nutricional do predador às diferentes presas utilizadas naquela oportunidade.

Larvas alimentadas com ovos de $A$. kuehniella e suplementadas com Toxoptera sp. ou com Pinnaspis sp., ou ainda aquelas que receberam esses três tipos de presas, tiveram duração menor para o $3^{\circ}$ ínstar, seguidas por aquelas que receberam apenas ovos de A. kuehniella. A maior duração foi verificada com Toxoptera sp.. O efeito de diferentes dietas sobre a duração do 30 ínstar de C. cubana confirma os resultados obtidos por Moraes (1989), para essa espécie, por Awadallah et al. (1975), para C. carnea e por Krishnamoorthy \& Many (1982), para Chrysopa scelestes Banks.

Duração da Fase de Larva. Observou-se que dietas contendo ovos de A. kuehniella propiciaram menor duração da fase larval de C. cubana (Tabela 1). Quando alimentadas somente com ovos de A. kuehniella, tiveram duração de 12,7 dias. Esses resultados aproximaram-se das observações de Pasqualini (1975), que ao usar a mesma dieta para $C$. carnea, constatou uma duração média de 15 dias para a fase larval. Esse fato pode estar relacionado não somente às diferenças nas condições ambientais dos locais onde foram instalados os experimentos, como também à capacidade de cada espécie em aproveitar eficientemente os nutrientes providos em determinado tipo de presa.

Quando foi fornecido apenas Toxoptera sp. ou este em combinação com Pinnaspis sp., observou-se duração intermediária da fase larval (19,0 e 17,1 dias, respectivamente). Por outro lado, aquelas alimentadas apenas com
Pinnaspis sp. foram as que apresentaram o maior período larval (20,5 dias). É importante considerar que, em condições naturais, o predador deve encontrar vários tipos de presa, permitindo uma redução na duração desta fase. Assim, Pinnaspis sp. pode constituir-se em uma espécie de presa alternativa de grande importância para o desenvolvimento de $C$. cubana no campo. Entretanto, em laboratório, não deverá ser usada, pois interferirá negativamente nas fases subsequentes, conforme mostrado a seguir.

Duração da Fase de Pupa. As pupas oriundas de larvas alimentadas com ovos de A. kuehniella + Toxoptera sp., A. kuehniella + Pinnaspis sp., bem como com a combinação dessas três dietas, tiveram desenvolvimento mais rápido, em relação àquelas que receberam as demais presas (Tabela 1). Esses resultados demonstraram que a qualidade do alimento ingerido na fase larval afetou a duração da fase de pupa, concordando com Ribeiro (1988), para C. externa. Contrastaram, porém, com os resultados de Pasqualini (1975), Krishnamoorthy \& Many (1982), Tartarini (1983) e Letardi \& Caffarelli (1989), que demonstraram que a duração da fase pupal das espécies de crisopídeos estudadas, ( $C$. carnea e $C$. scelestes) não diferiu quando as larvas foram alimentadas com diferentes dietas.

A duração desta fase para larvas alimentadas com Toxoptera sp., não foi realizada, posto que nenhuma delas conseguiu atingir a fase de pupa. Desta forma, embora Toxoptera sp. deva ser importante para o desenvolvimento e manutenção de populações de $C$. cubana no campo, em laboratório não deverá ser utilizado isoladamente como presa, por não permitir o desenvolvimento desse predador além da fase de larva.

Período de Larva a Emergência do Adulto. Este período apresentou uma duração significativamente menor quando as larvas foram alimentadas apenas com ovos de $A$. kuehniella ou quando estes foram suplementados com Toxoptera sp. ou 
Pinnaspis sp. (Tabela 1), evidenciando que essas dietas foram qualitativamente adequadas, uma vez que a menor duração nas fases imaturas dos insetos geralmente caracteriza uma melhor adequabilidade alimentar. Entretanto, um prolongamento na duração desse período foi constatado para larvas alimentadas com Toxoptera $\mathrm{sp} .+$ Pinnaspis sp. Os valores médios obtidos para larvas alimentadas com ovos de A. kuehniella (25,7 dias) e com ovos de A. kuehniella + Toxoptera sp. (24,9 dias) foram inferiores àqueles verificados por Venzon \& Carvalho (1993), em condições semelhantes.

Viabilidade. Constatou-se, para o $1^{\circ}$ ínstar, uma viabilidade de $100 \%$, exceto para a dieta a base de Pinnaspis sp. (95\%) (Tabela 2). Observou-se que houve uma redução na viabilidade dos ínstares subsequentes, exceto para larvas alimentadas com ovos de $A$. kuehniella, que apresentaram uma viabilidade ou Pinnaspis sp. morreram antes de completar o 3 o ínstar. Tal fato pode ser atribuído a inadequabilidade nutricional dessas dietas, que se evidenciou em insetos do 3 o ínstar. Observações semelhantes foram feitas por Hydorn \& Whitcomb (1979), quando alimentaram Chrysopa rufilabris Burmeister com Tetranychus gloveri Banks (Acari: Tetranychidae), e por Ribeiro (1988), quando forneceu $T$. citricidus à $C$. externa.

A viabilidade da fase pupal foi maior quando as dietas fornecidas às larvas foram ovos de A. kuehniella isoladamente ou suplementados com Toxoptera sp. ou Pinnaspis sp. ou, ainda, a combinação dessas 3 dietas (Tabela 2). Convém ressaltar que ovos de A. kuehniella fornecidos isoladamente, apesar de permitirem uma viabilidade de 100 $\%$ da fase larval, propiciaram $75 \%$ de viabilidade da fase de pupa. Esse fato pode ser atribuído à necessidade de uma suplementação à essa dieta.

Tabela 2. Viabilidade das fases imaturas de Ceraeochrysa cubana em diferentes dietas.

\begin{tabular}{|c|c|c|c|c|c|}
\hline \multirow{3}{*}{ Tratamentos } & \multicolumn{4}{|c|}{ Viabilidade em \% } & \multirow{3}{*}{$\begin{array}{c}\text { Percentagem } \\
\text { de adultos } \\
\text { emergidos }\end{array}$} \\
\hline & \multicolumn{3}{|c|}{ Ínstares } & \multirow{2}{*}{$\begin{array}{c}\text { Fase de } \\
\text { Pupa }\end{array}$} & \\
\hline & $1^{\mathrm{o}}$ & $2^{-}$ & $3^{\circ}$ & & \\
\hline 1. A. kuehniella. & 100,0 & 100,0 & 100,0 & 75,0 & 75,0 \\
\hline 2. Toxoptera sp. & 100,0 & 95,0 & 31,6 & 0,0 & 0,0 \\
\hline 3. Pinnaspis sp. & 95,0 & 68,4 & 15,4 & 50,0 & 5,0 \\
\hline $\begin{array}{l}\text { 4. A. kuehniella }+ \\
\text { Toxoptera sp. } \\
\text { 5. A. kuehniella }+\end{array}$ & 100,0 & 80,0 & 87,5 & 100,0 & 70,0 \\
\hline $\begin{array}{l}\text { Pinnaspis sp. } \\
\text { 6. Toxoptera } \mathrm{sp} .+\end{array}$ & 100,0 & 100,0 & 95,0 & 100,0 & 95,0 \\
\hline $\begin{array}{l}\text { Pinnaspis sp. } \\
\text { 7. A. kuehniella }+ \\
\text { Toxoptera sp. + }\end{array}$ & 100,0 & 100,0 & 50,0 & 30,0 & 15,0 \\
\hline Pinnaspis sp. & 100,0 & 100,0 & 90,0 & 88,9 & 80,0 \\
\hline
\end{tabular}

de $100 \%$ nos 3 ínstares. Pasqualini (1975) trabalhando com C. carnea, obteve $73,5 \%$ de pupação com essa mesma dieta. A maioria das larvas alimentadas com Toxoptera sp. e/
De uma maneira geral, pode-se observar que ovos de A. kuehniella fornecidos isoladamente ou como suplemento, juntamente com Toxoptera sp. e/ou Pinnaspis sp., 
permitiram a obtenção do maior número de adultos, comprovando que muitas presas, são aceitas como alimento pelas larvas, apesar de não serem nutricionalmente adequadas ao desenvolvimento da espécie.

\section{Agradecimentos}

A Fundação de Amparo à Pesquisa do Estado de Minas Gerais, FAPEMIG, pelo auxílio financeiro para execução do trabalho.

\section{Literatura Citada}

Agnew, C.W., W.L. Sterling \& D.A. Deans. 1981. Notes on the Chrysopidae and Hemerobiidae onf eastern Texas with key for their identification. Southwest. Entomol. 4:1-20.

Adams, P.A. \& N.D. Penny. 1985. Neuroptera of the Amazon basin: Part IIa. Introduction and Chrysopini. Acta Amazônica 15: 413-479.

Awadallah, K.T., N.A.Abou-Feid \& M.F.S. Tawafik. 1975. Development and fecundity of Chrysopa carnea Stephens. Bull. Soc. Entomol. Égypte 59: 323-329.

Hydorn, S. \& W.H. Whitcomb. 1979. Effects of larval diet on Chrysopa rufilabris. Fla Entomol. 62: 293-298.

Krishnamoorthy, A. \& M. Mani. 1982. Feeding potential and development of Chrysopa scelestes Banks on Heliothis armigera (Hubn.) under laboratoy conditions. Entomon 7 : 385-388.

Letardi, A. \& V. Caffarelli. 1989. Impiego di uma dieta semi-artificiale allo stato liquido per l'allevamento di larve di Chrysoperla carnea (Stephens) (Planipennia: Chrysopidae). Redia 72: 192-203.

Moraes, J.C. 1989. Aspectos biológicos e seletividade de alguns acaricidas à Ceraeochrysa cubana (Hagen, 1861) (Neuroptera: Chrysopidae) em laboratório. Tese de mestrado, ESAL, Lavras, 86p.

Muma, M.H. 1957. Effects of larval nutrition on the life cycle, size, coloration, and longevity of Chrysopa lateralis Guer. Fla Entomol. 40: 5-9.

Obrycki, J.J., M.N. Hamid, A.S. Sjap \& A.C. Lewis. 1989. Suitability of corn insect pests for development and survival of Chrysoperla carnea and Chrysopa oculata (Neuroptera: Chrysopidae). Environ. Entomol. 18: 1126-1130.

Pasqualini, E. 1975. Prove di allevamento in ambiente condizionato di Chrysopa carnea Steph. (Neuroptera: Chrysopidae). Boll. Ist. Ent. Univ. Bologna 32: 291-304.

Ribeiro, M.J. 1988. Biologia de Chrysoperla externa (Hagen, 1861) (Neuroptera: Chrysopidae) alimentada com diferentes dietas. Tese de mestrado, ESAL, Lavras, $131 \mathrm{p}$.

Tartarini, E. 1983. Influenza di differenti metodi di allevamento larvale sullo sviluppo e sulla fecondità di Chrysoperla carnea (Stephens) (Neuroptera: Chrysopidae). Boll. Ist. Ent. Univ. Bologna 38: 1-24.

Tauber, C.A. 1974. Systematics of north american chrysopid larvae: Chrysopa carnea group (Neuroptera). Can. Entomol. 106: 1133 - 1153.

Venzon, M. 1991. Biologia de Ceraeochrysa cubana (Hagen, 1861) (Neuroptera: Chrysopidae) em diferentes dietas e temperaturas. Tese de mestrado, ESAL, Lavras, 122p.

Venzon, M. e Carvalho, C.F. 1993. Desenvolvimento larval, pré-pupal e pupal de Ceraeochrysa cubana (Hagen) (Neuroptera: Chrysopidae) em diferentes dietas e temperaturas. An. Soc. Entomol. Brasil 22: 477-483.

Recebido em 13/02/96. Aceito em 09/07/97. 